\title{
Anti-PD-LI F(ab) Conjugated PEG-PLGA Nanoparticle Enhances Immune Checkpoint Therapy
}

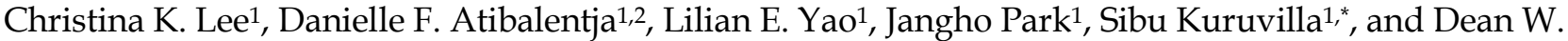 \\ Felsher ${ }^{\square}$ \\ 1. Division of Oncology, Departments of Medicine and Pathology, Stanford University School of Medicine, Stanford, CA, USA \\ 2. Division of Hematology, Department of Medicine, Stanford University School of Medicine, Stanford, CA, USA \\ ${ }^{*}$ Current location: Genentech, South San Francisco, CA, USA \\ $\bowtie$ Corresponding author: dfelsher@stanford.edu
}

(C) The author(s). This is an open access article distributed under the terms of the Creative Commons Attribution License (https://creativecommons.org/licenses/by/4.0/). See http://ivyspring.com/terms for full terms and conditions.

Received: 2021.07.31; Accepted: 2021.12.21; Published: 2022.01.16

\begin{abstract}
Background: Immune checkpoint therapies are effective in the treatment of a subset of patients in many different cancers. Immunotherapy offers limited efficacy in part because of rapid drug clearance and off-target associated toxicity. PEG-PLGA is a FDA approved, safe, biodegradable polymer with flexible size control. The delivery of immune checkpoint inhibitors such as anti-PD-L1 ( $\alpha$-PD-L1) via PEG-PLGA polymer has the potential to increase bioavailability and reduce immune clearance to enhance clinical efficacy and reduce toxicity.

Methods: The Fc truncated F(ab) portion of $\alpha-P D-L 1$ monoclonal antibody ( $\alpha-P D-L 1$ mAb) was attached to a PEG-PLGA polymer. $\alpha$-PD-LI $F(a b)-P E G-P L G A$ polymers were incubated in oil-in-water emulsion to form a $\alpha-P D-L 1$ F(ab)-PEG-PLGA nanoparticle ( $\alpha-P D-L 1$ NP). $\alpha$-PD-L1 NP was characterized for size, polarity, toxicity and stability. The relative efficacy of $\alpha-P D-L 1 ~ N P$ to a-PD-L1 mAb was measured when delivered either intraperitoneally (IP) or intravenously (IV) in a subcutaneous mouse colon cancer model (MC38). Antibody retention was measured using fluorescence imaging. Immune profile in mice was examined by flow cytometry and immunohistochemistry.

Results: Engineered Q-PD-L1 NP was found to have pharmacological properties that are potentially advantageous compared to $\alpha-P D-L 1$ mAb. The surface charge of $\alpha-P D-L 1$ NP was optimal for both tumor cell uptake and reduced self-aggregation. The modified size of $\alpha$-PD-LI NP reduced renal excretion and mononuclear phagocyte uptake, which allowed the NP to be retained in the host system longer. $\alpha-P D-L 1$ NP was non-toxic in vitro and in vivo. $\alpha-P D-L 1$ NP comparably suppressed MC38 tumor growth. $Q$-PD-LI NP appeared to elicit an increased immune response as measured by increase in germinal center area in the spleen and in innate immune cell activation in the tumor. Finally, we observed that generally, for both $\alpha-P D-L 1 ~ N P$ and $\alpha-P D-L 1 ~ m A b$, the IP route was more effective than IV route for tumor reduction.

Conclusion: $\alpha-P D-L 1$ NP is a non-toxic, biocompatible synthetic polymer that can extend $\alpha-P D-L 1$ antibody circulation and reduce renal clearance while retaining anti-cancer activity and potentially enhancing immune activation.
\end{abstract}

Key words: PD-L1, PEG-PLGA, MC38, Immunotherapy

\section{Introduction}

Checkpoint inhibitors that target CTLA-4 and PD-1/PD-L1 have exhibited success in the treatment of both solid and hematological malignancies leading to FDA approval [1-6]. However, only about $20-40 \%$ 
of patients show response to immunotherapy [7]. Current immunotherapy offers limited benefit due to poor tumor-specific distribution, rapid clearance (half-life of 24 hours to 3 days), and off-target distribution/toxicity [6, 8-12]. Approximately 3-40\% of injected monoclonal antibodies $(\mathrm{mAb})$ reach the solid tumor tissue [13, 14]. This is in part because mAbs are rapidly cleared through glomerular filtration in the kidneys due to their size being less than $10 \mathrm{~nm}$ (mAb average size, 5.2-7.1 nm). Fatal immune-related adverse effects range from 15-70\% depending on the regimens [15-18]. To address these pharmacologic limitations, various nanoparticles have been developed that can potentially improve $\mathrm{mAb}$ delivery and retention while reducing toxicity [19].

We investigated whether the checkpoint inhibitor, a-PD-L1 mAb, loaded onto a biodegradable polymer could be used to enhance their pharmacologic properties. Antibodies are composed of an antigen binding fragment $\mathrm{F}(\mathrm{ab})$ region and a constant Fc region [20]. The Fc portion of mAb results in immune clearance [21-23]. This elicits immune-related toxicity such as colitis, hepatitis, pneumonitis [24-27]. We reasoned that we may reduce immune clearance and toxicity by loading the $\mathrm{F}(\mathrm{ab})$ of $\mathrm{a}-\mathrm{PD}-\mathrm{L} 1 \mathrm{mAb}$ onto a poly(ethylene glycol)-poly(lactic-co-glycolic acid) (PEG-PLGA) delivery system.

Nanoparticle coating with a water-soluble, "stealth-like" polymer such as PEG has been shown to protect the payload from rapid clearance and enhance the a-PD-L1 mAb biodistribution properties [28, 29]. PEG and PLGA are FDA-approved nanoparticle carriers that exhibit versatile functionality, synthetic feasibility, and have already been shown to improve pharmacokinetics, tumor distribution, and safety profile of drug payloads [30-34]. Passive targeting is facilitated by the enhanced permeation and the retention (EPR) effect from the leaky vasculature and compromised lymphatic system of the tumor tissue [35]. Active targeting is achieved by the display of tumor-specific targeting ligands or antibodies [35]. Hence, we inferred that we may enable tumor-specific delivery of the payload through both passive and active targeting to tumor tissue.

We hypothesized that a-PD-L1 NP will exhibit improved therapeutic efficacy as a result of employing the pharmacokinetics of the PEG-PLGA nanoparticles and removing the Fc portion of a-PD-L1 $\mathrm{mAb}$. Our results show that a-PD-L1 NP improves the circulation time of $\mathrm{a}-\mathrm{PD}-\mathrm{L} 1 \mathrm{mAb}$ via the modification of its pharmacologic properties and maintains the anti-tumor activity in a MC38 colorectal tumor model. This proof-of-concept study establishes that a-PD-L1 $\mathrm{F}(\mathrm{ab})$-conjugated PEG-PLGA nanoparticles may be useful as a platform technology to be investigated for other therapeutic mAbs. We also highlight the importance of characterizing both NP stability and route of delivery.

\section{Results}

\section{Synthesis and characterization of $\alpha-P D-L 1$ F(ab)-PEG-PLGA nanoparticles}

We generated a-PD-L1 F(ab)-PEG-PLGA nanoparticles (a-PD-L1 NP) intended to reduce premature clearance and off-target immune-mediated toxicity (Figure 1A). Since the $\mathrm{FC}$ region of the $\mathrm{a}-\mathrm{PD}-\mathrm{L} 1 \mathrm{mAb}$ is recognized by the Fc receptors on immune compartments leading to off-target immune activation, we first fragmented the a-PD-L1 antibodies into $\mathrm{F}(\mathrm{ab})$ and $\mathrm{Fc}$ portions as confirmed by the molecular weights measured by MALDI-TOF (Figure 1B). We then loaded the $\mathrm{F}(\mathrm{ab})$ portion of the a-PD-L1 mAb onto PEG-PLGA to enhance pharmacologic properties. A maleimide linker functionalized to the end of PEG-PLGA polymers was used to conjugate to the free thiol groups exposed on the $\mathrm{F}(\mathrm{ab})$ after reduction. The a-PD-L1 NP was formed by using a standard oil-in-water emulsion procedure and the geometry of the nanoparticles was confirmed using Dynamic Light Scattering (DLS) and Zeta Potential measurements (Figure 1C). After attachment to PEG-PLGA polymers, the geometry of the antibodies was increased to above the limit for renal excretion of greater than or equal to 10nm (a-PD-L1 NP, average size $260.2 \mathrm{~nm}$ ) with a surface charge that falls between $-10 \mathrm{mV}$ and $10 \mathrm{mV}$ (average peak $5.3 \mathrm{mV}$ ). Both the size and charge of the nanoparticle fell in ranges that are favorable for cellular uptake and avoid self-aggregation between the nanoparticles [36, 37]. The modified size and surface charge are intended to minimize the rapid clearance of the antibodies after intravenous (IV) or intraperitoneal (IP) injection while staying below $500 \mathrm{~nm}$ to avoid unintentional uptake by the mononuclear phagocytic system (MPS) [38]. Our results indicate that the a-PD-L1 NP are synthetically feasible with amenable pharmacokinetics.

We next characterized the stability of a-PD-L1 NP over time. The size of PEG-PLGA (empty NP) and a-PD-L1 NP were measured over 10 weeks with the DLS method. The size of empty NP remained consistent over the period of 10 weeks (PEG-PLGA, average size $=199.4 \mathrm{~nm}$ ). However, the size of a-PD-L1 NP fluctuated by up to 21 percent over 2 weeks and gradually declined to a size comparable to that of empty NP by 10 weeks (Figure 2A). We also noted that the size of a-PD-L1 NP varied widely between six batches made at different time points for 
separate in vivo experiments. The size ranged from $147-450.1 \mathrm{~nm}$ as measured by DLS and transmission electron microscopy (TEM) while the size of the empty NP varied from $142.1 \mathrm{~nm}$ to $294.55 \mathrm{~nm}$ (Figure S1). This data suggests there is a change in stability of a-PD-L1 NP over time. For all our experiments, we manufactured new nanoparticles and validated their size.

\section{Toxicity in vitro and in vivo}

Next, we evaluated the toxicity of a-PD-L1 NP using in vitro and in vivo model systems. Murine colon tumor cells, MC38, were cultured in complete media with either a-PD-L1 mAb, empty NP, or a-PD-L1 NP. Total cell number was assessed after two doubling

\section{A}

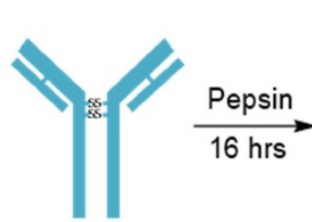

anti PD-L1

$\mathrm{mAb}$ time (doubling time measured appx. 13hours). The cell numbers did not differ significantly between the three groups and the cultures doubled at a consistent rate (Figure 2B). As expected, a-PD-L1 $\mathrm{mAb}$ had no direct effects on tumor cell viability. We also examined the effects of a-PD-L1 NP in vivo. Control mice without tumor exposure were injected intraperitoneally with control IgG, a-PD-L1 mAb, or a-PD-L1 NP. We did not find any significant changes between the treatment groups with regards to mice weight, spleen size, and behavior over the course of two months (Figure 2C). We conclude that a-PD-L1 $\mathrm{NP}$ has no apparent toxicity in vitro or in vivo.

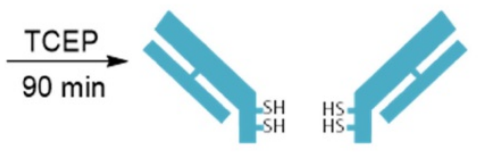

anti PD-L1 F(ab)

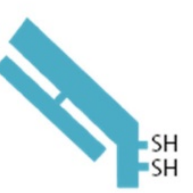<smiles>CC(C)OCC(C)(C)CNC(=O)CCN1C(=O)C=CC1=O</smiles>

anti PD-L1 $F(a b)$

maleimide-PEG-PLGA

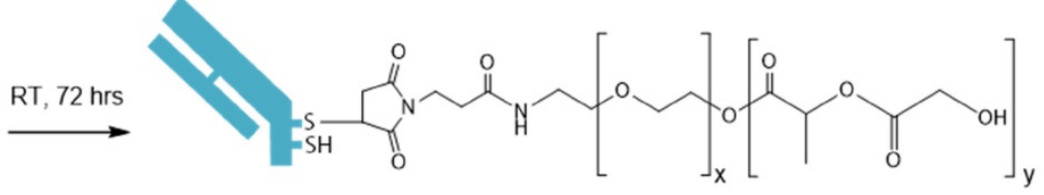

anti PD-L1 F(ab)-PEG-PLGA

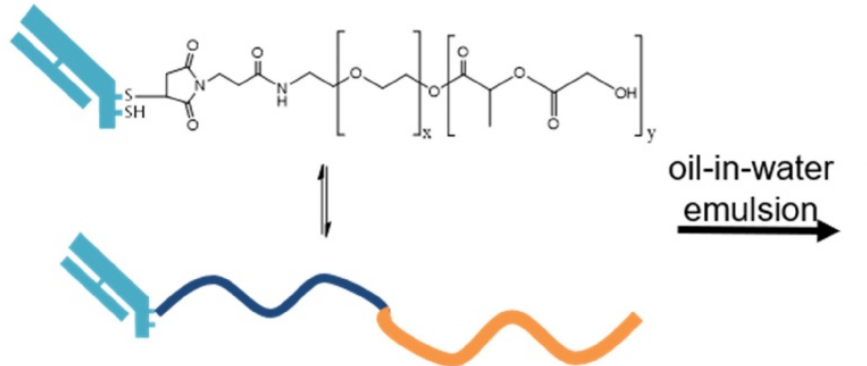

anti PD-L1 F(ab)-PEG-PLGA, DMSO

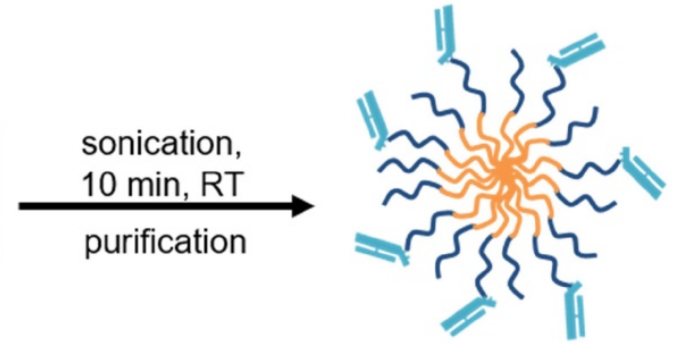

anti PD-L1 NP 
B

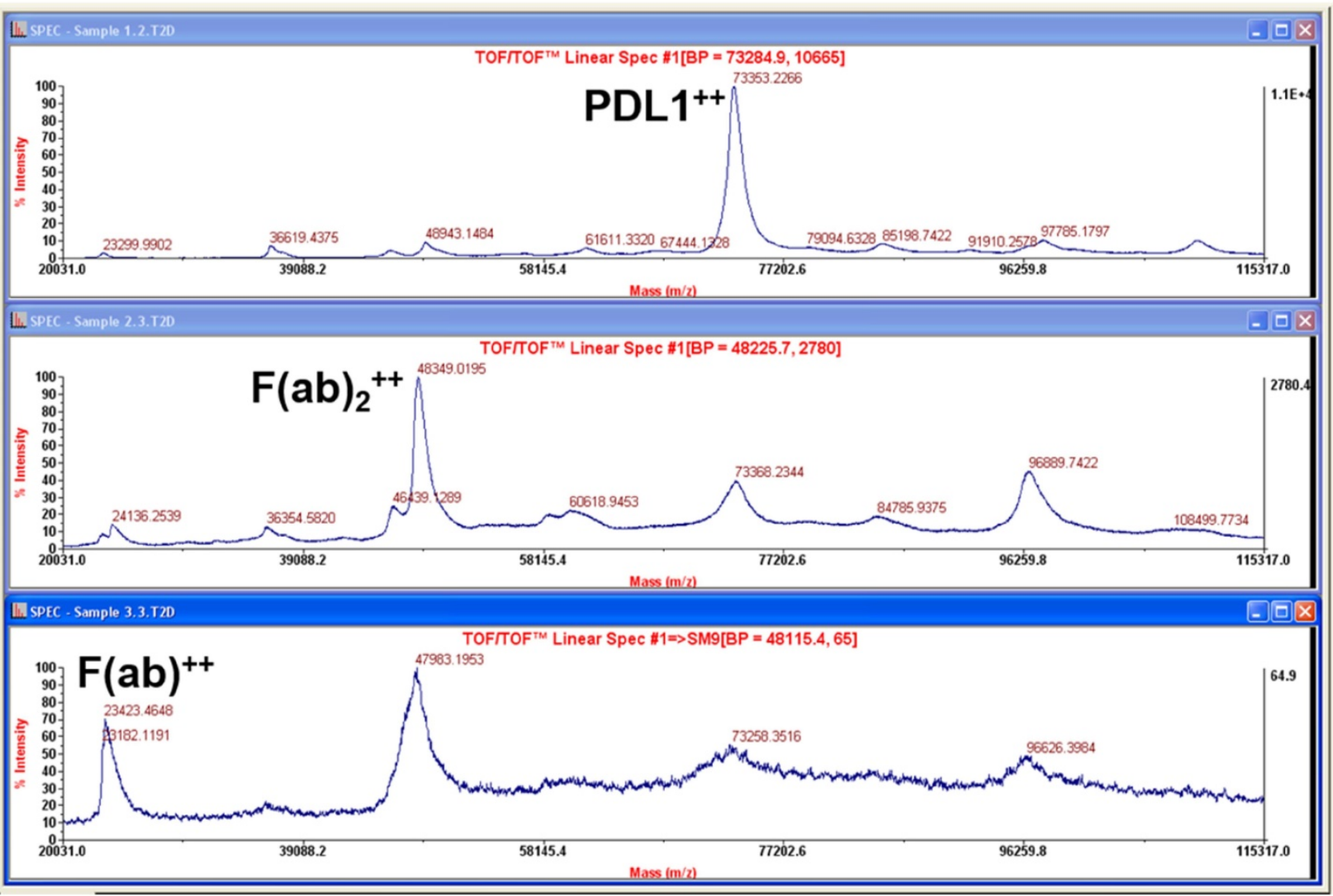

C

Hydrodynamic Diameter

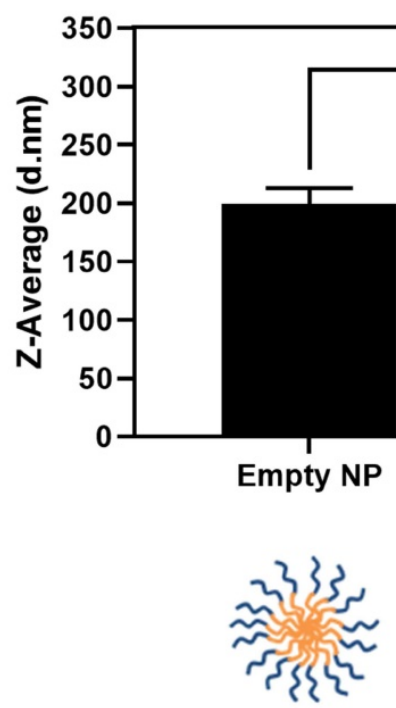

Surface Charge
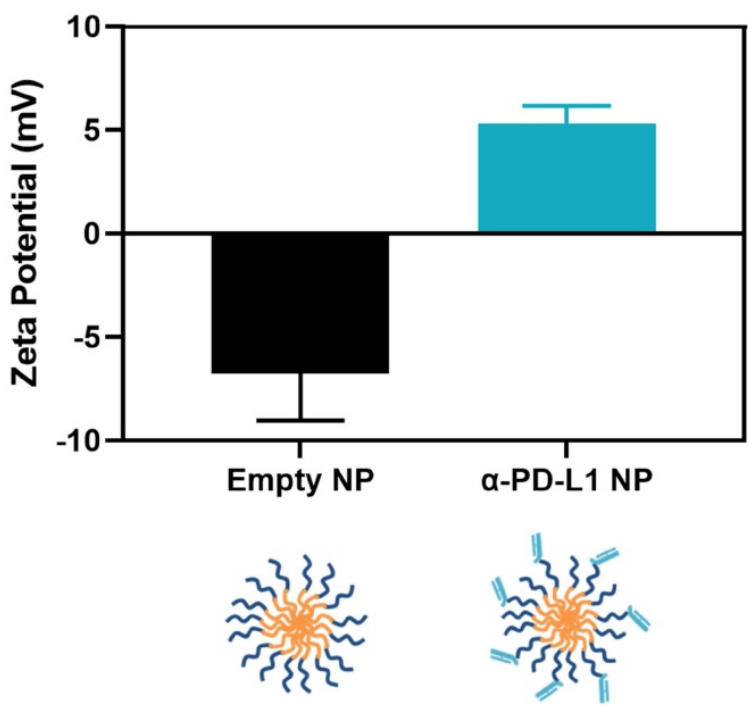

Figure 1. Nanoparticle characterization. (A) Schematics of $\alpha-P D-L 1$ F(ab)-PEG-PLGA synthesis. The Fc portion of the antibody is detached to reduce immune clearance and the remaining $\mathrm{F}(\mathrm{ab})$ portion is attached to PEG-PLGA to increase nanoparticle size in order to prevent antibody from premature clearance. PLGA is used to control the degradation rates or drug release rates. Water-soluble synthetic polymer, PEG, is used as a protein carrier, to reduce the immunogenicity of the conjugated proteins. (B) MALDI-TOF of $\alpha$-PD-L1 Fragmentation. MALDI-TOF of $\alpha-P D-L 1$ Fragmentation shows presence of both $F(a b)$ and $F(a b)_{2}$. The presence of $F(a b)_{2}$ is not relevant in the next antibody-to-polymer conjugation step since $\mathrm{F}(\mathrm{ab})_{2}$ lacks the free thiol groups exposed on $\mathrm{F}(\mathrm{ab})$ that is necessary for conjugation with maleimide linker on PEG-PLGA polymer.

The filtration step following the conjugation step eliminates any free antibody fragments that did not bind to polymers. (C) Size and surface charge of the empty NP ( $=16$ ) and a-PD-LI F(ab)-PEG-PLGA ( $\mathrm{n=27)}$ measured by 90Plus Particle Size Analyzer, Zetasizer Nano ZS90 and 90Plus Zeta Potential Analyzer. 
A

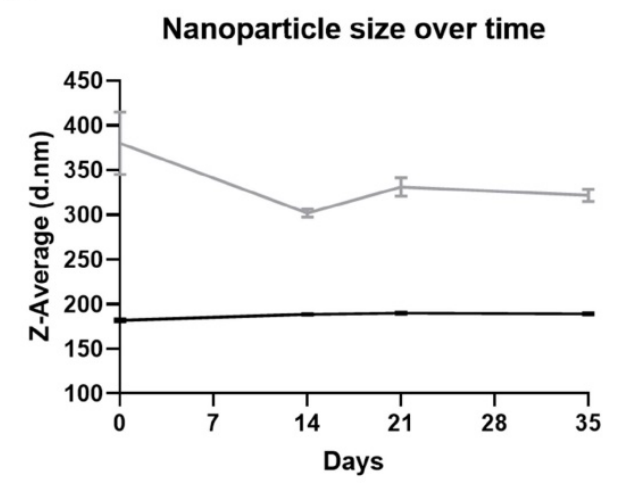

C

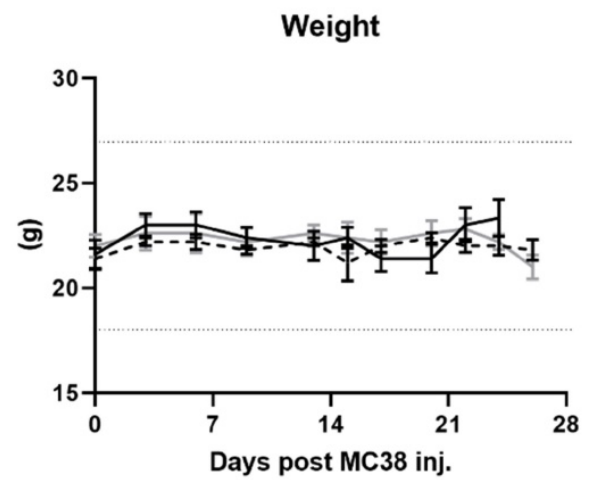

B

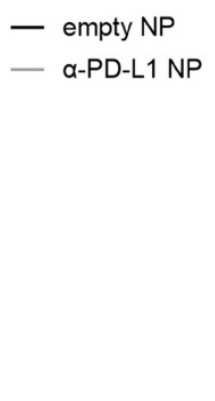

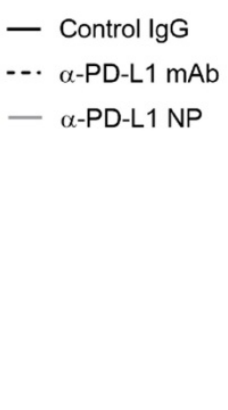

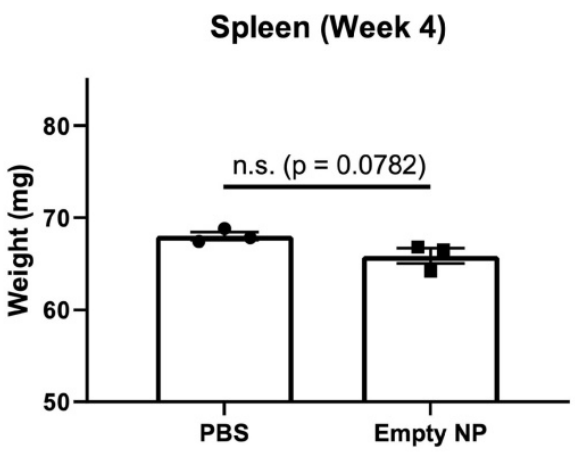

Figure 2. Nanoparticle Stability and Toxicity. (A) Size of empty NP and a-PD-L1 NP were tracked over time using Zetasizer Nano ZS90. ( $\mathrm{n}=3$ in each group). (B) Cell number of MC38 were counted 26 hours after culture with $10 \mu \mathrm{g} / \mathrm{ml} \alpha-P D-L 1 \mathrm{mAb}, \alpha-P D-L 1 \mathrm{NP}$, or empty NP. (C) Body weight of healthy mice that received control lgG, $\alpha-P D-L 1$ mAb, or $\alpha-P D-L 1$ NP were recorded every $3-4$ days $(n=3 ; 200 \mu g / m i c e, 3$ injections over 9 days; left). $20 \%$ weight loss is a criterion for euthanasia (dotted line above and below). Spleen weight of healthy mice that received either PBS or empty NP was measured at 4 weeks post treatment ( $\mathrm{n}=3$; right).

\section{Extended circulation time of immune checkpoint inhibitor}

We examined the kinetics and distribution of a-PD-L1 NP in vivo. A Cy5 dye was used to label a-PD-L1, a-PD-L1 F(ab) 2, a-PD-L1 F(ab), empty NP, and a-PD-L1 NP and achieved equivalent fluorescent labeling (data not shown). The fluorescently-labeled agents were injected intravenously at equivalent $\mathrm{Cy} 5$ concentration into NSG mice, a commonly employed mouse model for fluorescence imaging due to their white coat. Mice were monitored via fluorescence imaging at $0,4,8$, and 24 hours. The organs of mice that received a-PD-L1 F(ab)-PEG-PLGA had higher expression of fluorescence after 24 hours compared to those that received other regimens. The majority of the fluorescent signal disappeared in the four control groups in the liver, spleen, and kidney while a strong signal was maintained in the a-PD-L1 NP group (Figure 3). Thus, the size modification obtained by attaching the $\mathrm{F}(\mathrm{ab})$ portion of the immune checkpoint inhibitor to PEG-PLGA allowed the nanoparticles to persist longer in recipient mice.

\section{Route of drug administration}

The route of drug administration can influence therapeutic activity [39, 40]. We introduced a-PD-L1 NP either through tail vein (IV) or intraperitoneal (IP) to mice orthotopically transplanted with the MC38 tumor cell line. We found that tumor bearing mice that received a-PD-L1 NP through the IP injection had significantly reduced tumor growth compared to tumor bearing mice that received a-PD-L1 NP IV (Figure 4A). Intratumoral (IT) injection of immunotherapies is another route of administration commonly employed in medical research [41]. We compared the tumor growth curve in mice treated with a-PD-L1 NP IT, a-PD-L1 NP IP, IgG IP and found that mice treated with a-PD-L1 NP IT and IP exhibited delayed tumor growth compared to mice treated with IgG IP (Figure S2). However, there was significant variation within the a-PD-L1 NP IT group, which led us to focus on a-PD-L1 NP IP for subsequent studies. Our findings suggest that IP versus IV injection may improve the efficacy of a-PD-L1 NP. 


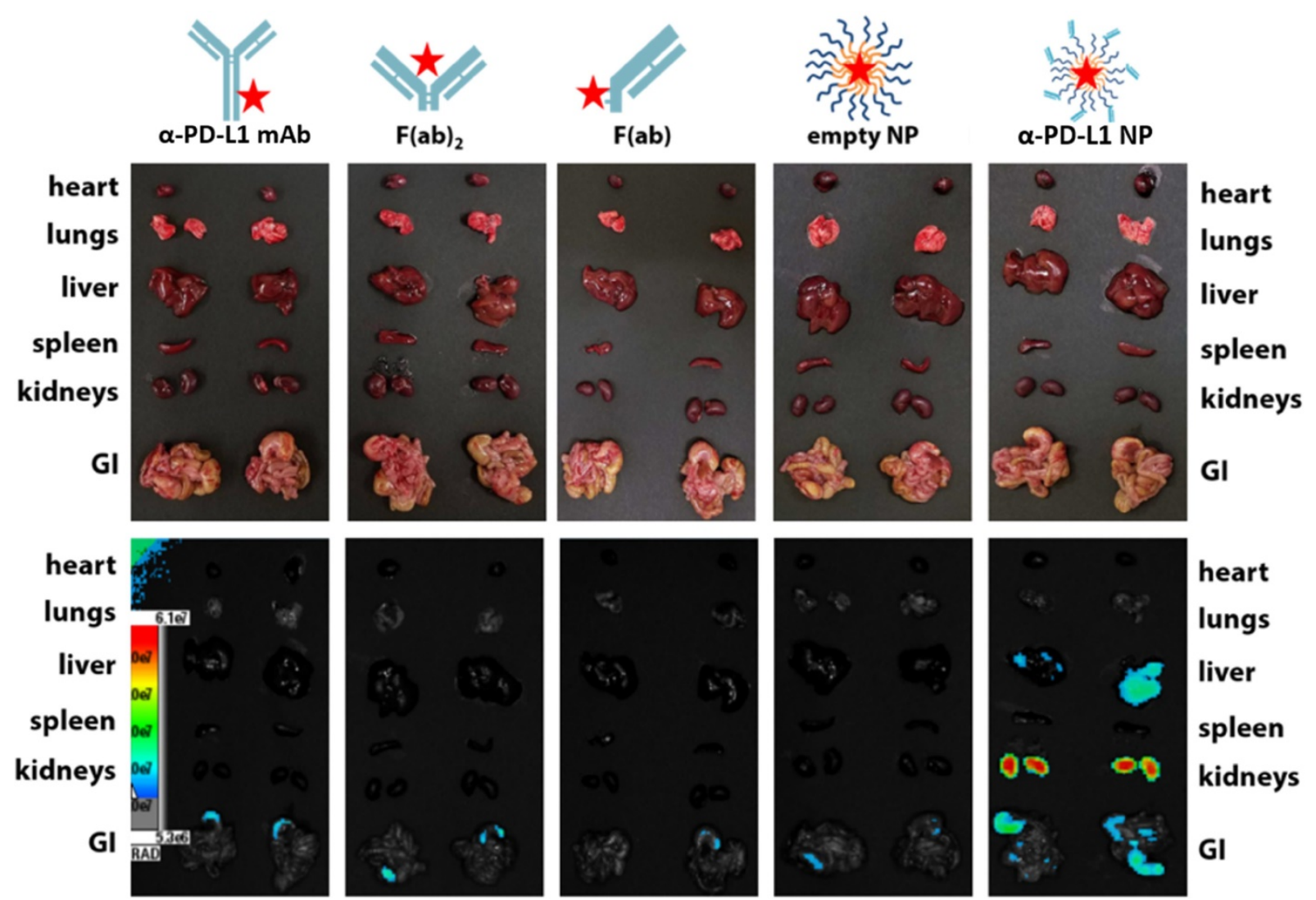

Figure 3. In vivo retention of intravenously injected NP compared to $\alpha-P D-L 1$ mAb. Cy5 dye tagged $\alpha-P D-L 1$ mAb, F(ab)2, F(ab), empty NP, and $\alpha-P D-L 1 ~ N P$ were injected into NSG mice IV and fluorescence in different organs was traced 24 hours post injection ( $500 \mu g$ of $\mathrm{mAb}$ and an equivalent amount of NPs were injected to mice).

\section{Therapeutic efficacy}

We compared the therapeutic efficacy of a-PD-L1 NP to a-PD-L1 mAb. MC38 tumors were grown $\mathrm{SC}$ in syngeneic $\mathrm{C} 57 \mathrm{BL} / 6 \mathrm{~J}$ mice which were then treated IP with either $\mathrm{a}-\mathrm{PD}-\mathrm{L} 1 \mathrm{mAb}$ or $\mathrm{a}-\mathrm{PD}-\mathrm{L} 1$ $\mathrm{NP}$ and tumor volume was assessed after 3 weeks. Tumor volumes in mice that received either a-PD-L1 $\mathrm{mAb}$ or $\mathrm{a}-\mathrm{PD}-\mathrm{L} 1 \mathrm{NP}$ were significantly smaller (mean tumor vol. $42.1 \mathrm{~mm}^{2}$ and $92.3 \mathrm{~mm}^{2}$, respectively) compared to tumor volumes in mice that received the control IgG (mean tumor vol. $513.2 \mathrm{~mm}^{2}$ ). There was no significant difference in tumor size between mice that received a-PD-L1 NP and a-PD-L1 mAb at day 20 post tumor cell injection (Figure 4B). We infer that a-PD-L1 NP and a-PD-L1 mAb exhibit similar anti-tumor activity in vivo.

\section{Changes in host immune activation}

Blocking PD-L1 on myeloid and lymphoid cells has been found to be important for responses to checkpoint blockade therapies [42-45]. We noted that treatment with a-PD-L1 mAb and a-PD-L1 NP both increased spleen size in mice compared to control mice (a-PD-L1 mAb and a-PD-L1 NP, mean spleen weight at $156.1 \mathrm{mg}$ and $115.5 \mathrm{mg}$, respectively, Figure $4 \mathrm{C}$; healthy mice, mean spleen weight at $68 \mathrm{mg}$, Figure 2C). We next measured the number and area of germinal centers (GC) in the spleen associated with activated $\mathrm{CD}^{+} \mathrm{T}$ cells and $\mathrm{B}$ cells [46-48]. Immunohistochemistry staining of CD4 and CD19 was used to estimate the GC number and area in the spleens just before the appearance of splenomegaly at 3 weeks post MC38 injection. The mice that received $\mathrm{a}-\mathrm{PD}-\mathrm{L1} \mathrm{mAb}$ and $\mathrm{a}-\mathrm{PD}-\mathrm{L} 1 \mathrm{NP}$ exhibited no significant difference in GC number (IgG, 23; a-PD-L1 $\mathrm{mAb}, 20.7$; a-PD-L1 NP, 20.7) and area (a-PD-L1 $\mathrm{mAb}, 246,235.8 \mu \mathrm{m}^{2}$; $\left.\mathrm{a}-\mathrm{PD}-\mathrm{L} 1 \mathrm{NP}, 173,370.6 \mu \mathrm{m}^{2}\right)$, and had comparable $\mathrm{CD} 4^{+} \mathrm{T}$ cell and $\mathrm{B}$ cell numbers at 3 weeks post MC38 injection (Figure 5A). Thus, $a-P D-L 1 ~ N P$ and a-PD-L1 mAb appear to induce similar immune activation early in the disease course.

We proceeded to examine the immune phenotype of cells in the spleen and tumor using multi-parameter flow cytometry. While not statistically significant, mice that received a-PD-L1 $\mathrm{NP}$ versus a-PD-L1 mAb showed an increase in the proportion of $\mathrm{CD}^{+} \mathrm{T}$ cells (26.8 versus 18.9 percent) and B cells (83.1 versus 59.5 percent) in the spleen, and a higher frequency of tumor infiltrating MHCII ${ }^{+}$Ly6C $/ \mathrm{G}^{+} \mathrm{F} 4 / 80^{+}$macrophages (18.2 versus 11.7 percent), MHCII-Ly6C/ $\mathrm{G}^{+} \mathrm{CD} 11 \mathrm{~b}^{+}$neutrophils (7.3 versus 2.4 percent) and $\mathrm{NK} 1.1^{+} \mathrm{CD} 49 \mathrm{~b}^{+} \mathrm{NK}$ cells ( 2.5 versus 0.5 percent) in the tumor at 4 weeks post MC38 injection (Figure 5B). No differences were 
found in CD4+ T cells in the spleen (a-PD-L1 mAb, 65.4 percent; a-PD-L1 NP, 61.9 percent) or tumor (a-PD-L1 mAb, 13.9 percent; a-PD-L1 NP, 13.5 percent) (Figure 5C). Treatment with a-PD-L1 NP demonstrated an immune profile suggestive of immune activation as indicated by higher frequencies of $\mathrm{CD}^{+} \mathrm{T}$ cells and B cells in the spleen, and tumor infiltrating inflammatory macrophages, neutrophils, and mature NK cells $[49,50]$.

A

Tumor volume

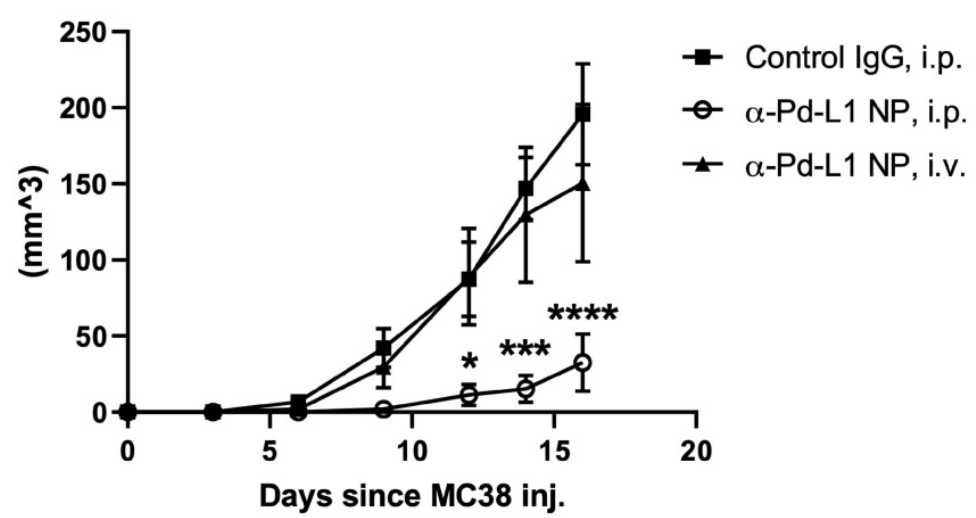

B

Tumor volume

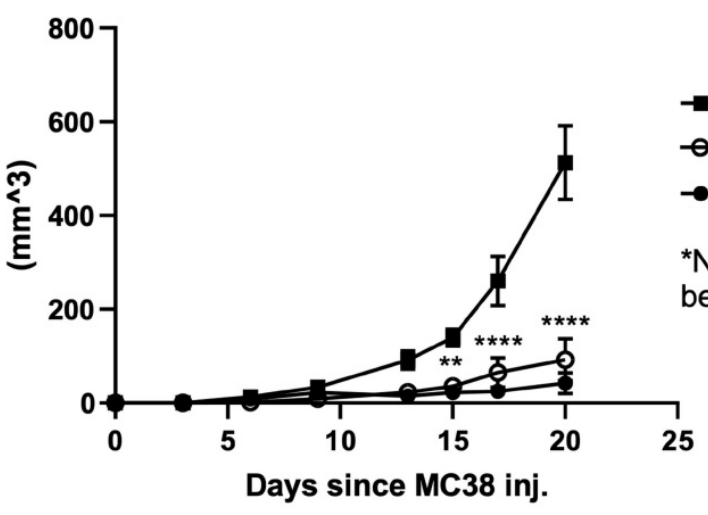

C

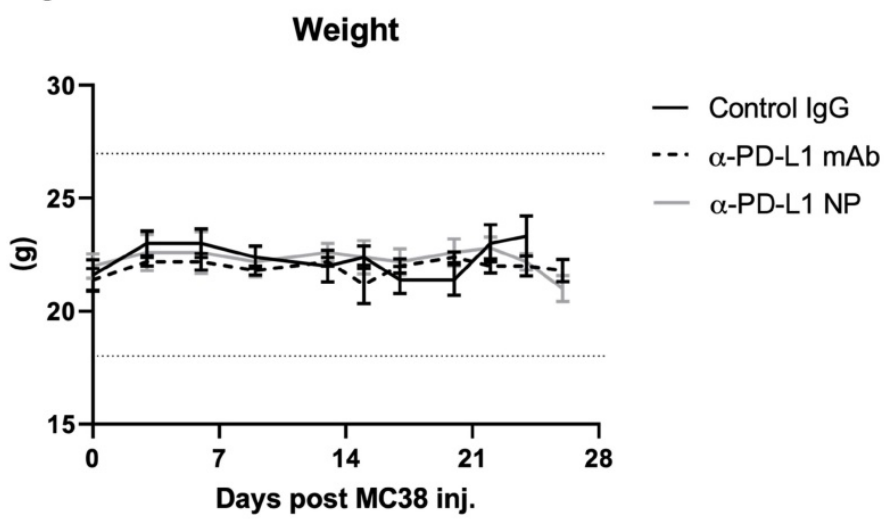

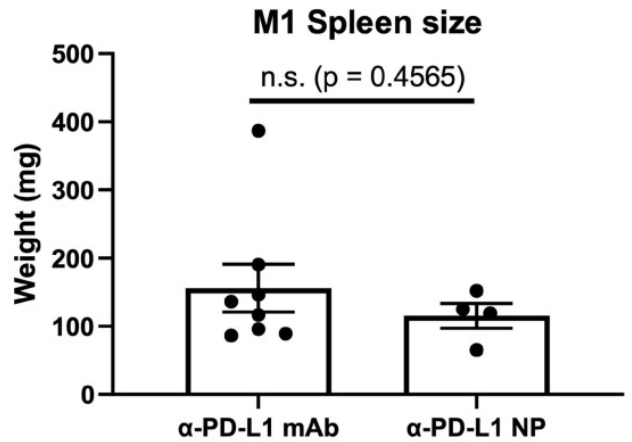

Figure 4. In vivo effects of injected NP based on route of administration. (A) Tumor volumes were measured in mice that received $\alpha-P D-L 1$ NP IP ( $=5$ ) versus IV $(n=4)$ until the first mouse reached endpoint as dictated by protocol. Control lgG $(n=3)$ was injected IP as standard practice $(200 \mu g / m i c e, 3$ injections over 9 days). (B) Tumor volumes between mice that received control IgG, $\alpha-P D-L 1$ mAb, or a-PD-LI NP were monitored every 3-4 days post MC38 injection for 3 weeks ( $n=5$ in each group; $200 \mu \mathrm{g} / \mathrm{mice}, 3$ injections over 9 days). (C) Body weight of mice that received control lgG, a-PD-L1 mAb, or a-PD-L1 NP IP were monitored every 3-4 days post MC38 injection for 24-26days ( $n=5$ in each group; left). Spleen weight was measured at the time mice were euthanized (right). 


\section{Discussion and Conclusion}

Various antibody conjugated nanoparticles are currently being explored to enhance targeted therapies [51]. Polymeric NPs are preferred for their easy size modification and biodegradability [52-54]. However, immune checkpoint therapies using pegylated nanoparticles have shown limited benefit in overall survival and increased toxicity in patients [55, 56]. Stability and drug efficacy of polymeric NP based therapies depend on the carrier type and strategies for antibody conjugation $[57,58]$.

The work described herein is a proof-of-concept study investigating the feasibility of attaching a-PD-L1 F(ab) fragments onto PEG-PLGA polymers to improve the efficacy of a-PD-L1 therapy. Our study indicates we can generate a nanoparticle with size and surface charge favorable for cellular uptake while avoiding nanoparticle aggregation, premature clearance, and MPS uptake. Further, a-PD-L1 NP significantly increased antibody retention in vivo and did not appear to have toxicity. We noted a-PD-L1 $\mathrm{F}(\mathrm{ab})$ attached to PEG-PLGA is prone to degradation and clustering $[20,59]$. Due to its nano-range size, a small change can result in significant size variation between batches and within the same batch over time. We found it was important to characterize a-PD-L1 NP immediately before use to reduce this potential confounding factor. Taken as a whole, our results suggest that PEG-PLGA mediated delivery of a-PD-L1-F(ab) may be a strategy to extend a-PD-L1 antibody retention and reduce toxicity. This method may be useful for other monoclonal antibody therapies.

Particle filtration in spleen, liver, and kidney increases with size. However, the maximal filtration found in the spleen is for particles sized $400 \mathrm{~nm}$ and above [60], liver for $107 \mathrm{~nm}$ and above [61], and kidney for 10nm and above [62]. The average size of our a-PD-L1-PEG-PLGA nanoparticle is $260.2 \mathrm{~nm}$. As expected, we found no trace of our engineered NPs in the spleen, a reduced expression in the liver, and the strongest expression in the kidney at 24 hours post injection. The reason why the empty nanoparticle (PEG-PLGA, average $199.4 \mathrm{~nm}$ ) was not similarly identified in the liver and kidney compared to the a-PD-L1 NP is unclear and requires more in-depth analysis. We suspect that the dual function of direct binding to PD-1/PD-L1 as well as slight differences in NP size may introduce different pharmacodynamics.

A

$\alpha-P D-L 1$ mAb
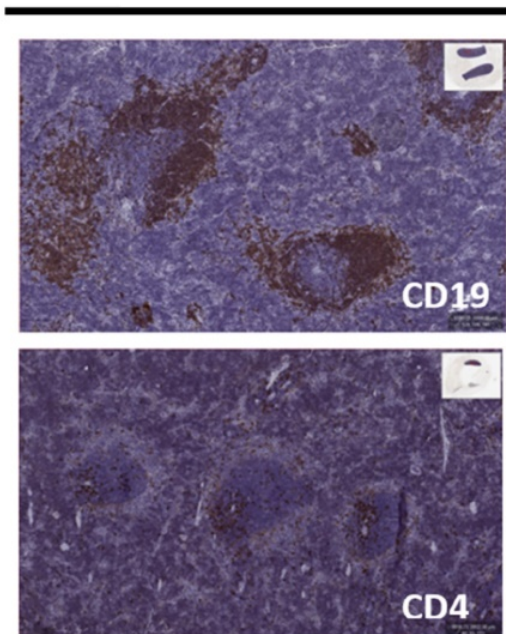

a-PD-L1 NP

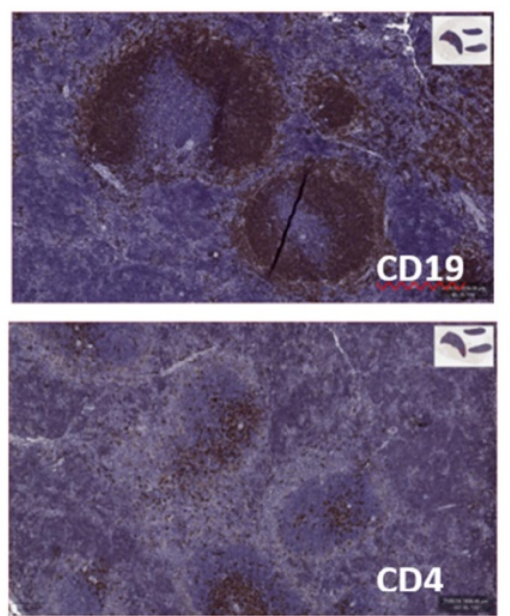

Number of Germinal Center (week 3)

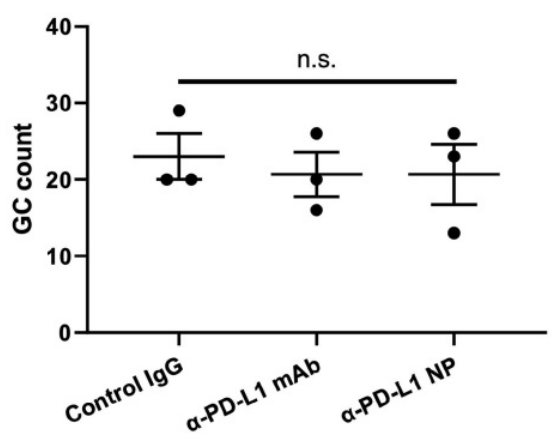

Estimation Plot (Area, $\mu \mathrm{m}^{2}$ )

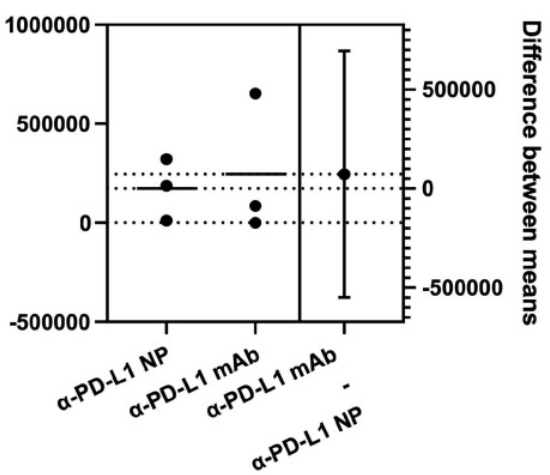


B

Spleen CD4

Spleen CD8

Spleen B cells
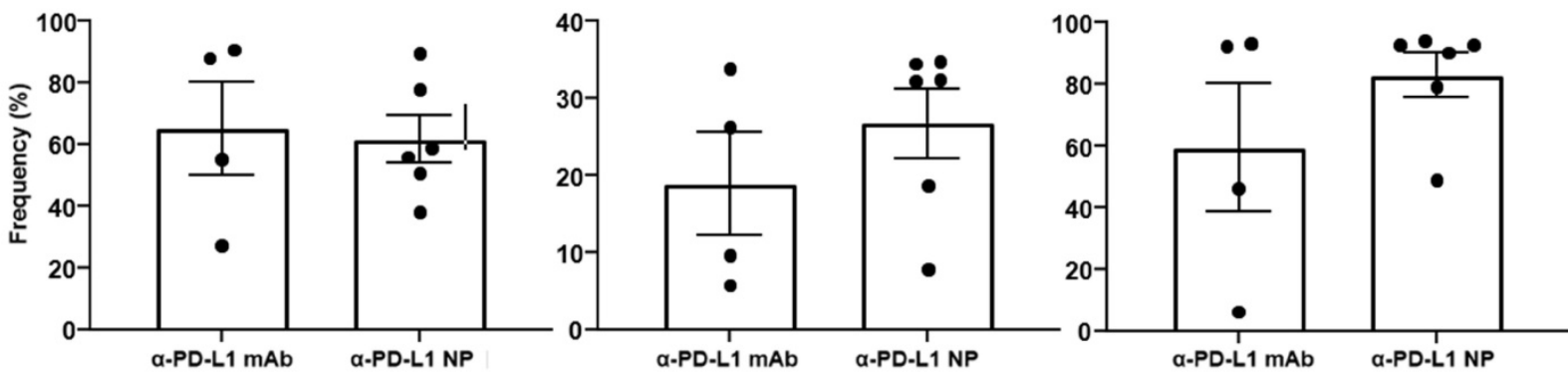

Spleen classical macrophage

Spleen Neutrophils

Spleen NK cells
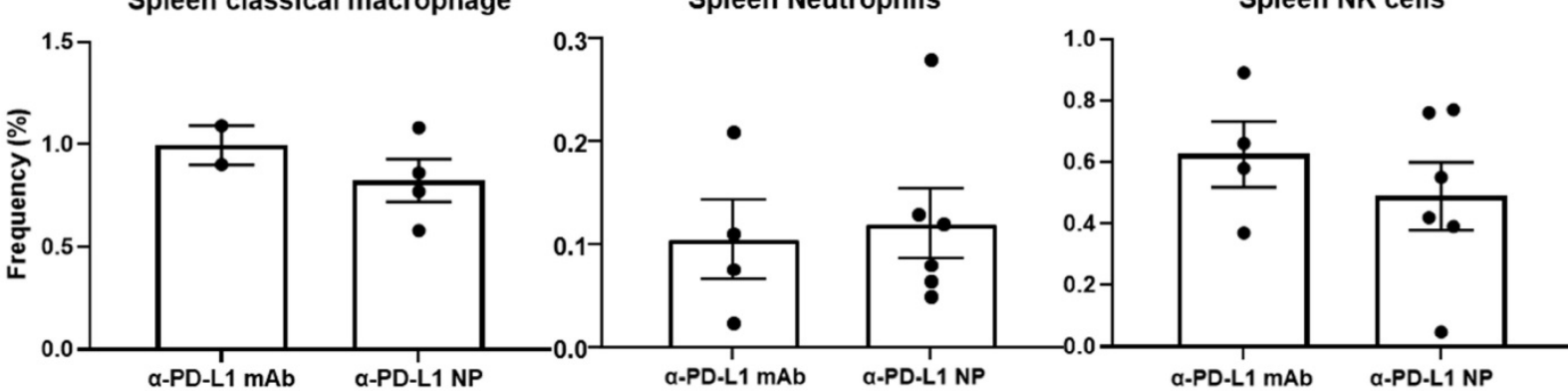

C

Tumor CD4

Tumor CD8

Tumor B cells
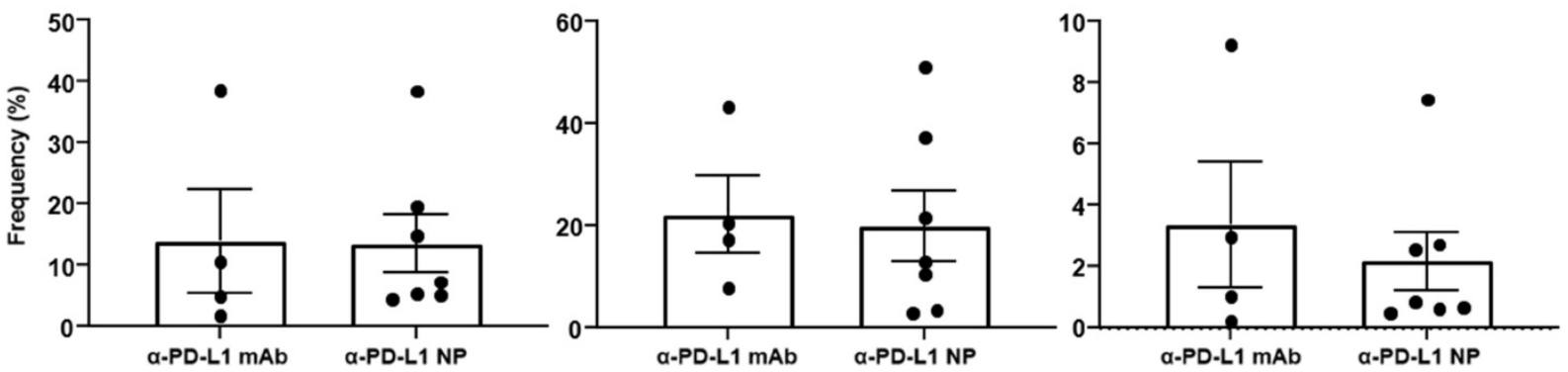

Tumor classical macrophages

Tumor Neutrophils

Tumor NK cells
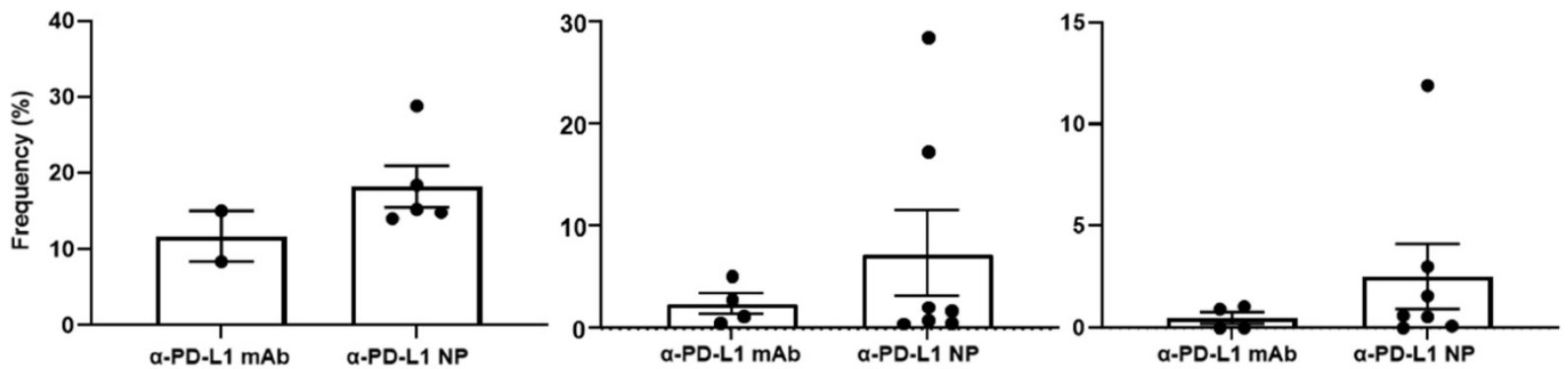

Figure 5. Change in immune profile over time. (A) IHC of CD4 and CD 19 were used to identify the GC region and quantify the number of GC in the spleen at 3 weeks post MC38 injection ( $n=3$ in each group). Flow cytometry was used for immune profiling of spleen (B) and tumor (C) of mice that received Q-PD-LI mAb or Q-PD-LI NP 4 weeks post MC38 injection.

We found that the route of drug administration is an important factor in a-PD-L1 NP efficacy. Antibodies are commonly injected IV into the host circulatory system to allow rapid target recognition through leaky vasculature in cancer. However, our a-PD-L1 NP exhibited significantly improved efficacy when delivered IP compared to IV. This may be because a-PD-L1 NP that is introduced directly into systemic circulation risks being entrapped in the liver and lung [63-65]. In addition, a-PD-L1 NP that is injected IP must pass through the lymphatic system before entering the circulatory system. a-PD-L1 NP 
that travels through the lymphatic system can interact and educate immune cells in the lymphatic system before tumor site entry $[66,67]$. Directly injecting a-PD-L1 NP to tumor (IT) had an effect but the mass of tumor may have been too small for the therapy to have a more meaningful result. Additional optimization different from our established treatment model may yield more consistent efficacy. Overall, the delivery of a-PD-L1 NP IP versus IV may be more efficacious.

We found a-PD-L1 mAb and a-PD-L1 NP were associated with similar immune activation. a-PD-L1 $\mathrm{NP}$ had higher frequencies of $\mathrm{CD} 8^{+} \mathrm{T}$ cells and $\mathrm{B}$ cells in the spleen and higher frequencies of inflammatory macrophages, neutrophils, and NK cells in the tumor. These changes have previously been shown to be favorable in the clinical setting $[68,69]$. However, we did not see evidence for enhanced efficacy in mice treated with a-PD-L1 NP. This may be because the immune activation observed may not be sufficient to confer increased tumor regression. Alternatively, the MC38 tumor assay we used was not sensitive enough to detect changes in efficacy when compared to the a-PD-L1 mAb. More in-depth evaluation of the impact of tumor heterogeneity and the effect of immunotherapy on tumor microenvironment may also yield a better understanding of the phenomenon we observed. Regardless, we conclude that a-PD-L1 $\mathrm{NP}$ demonstrated a comparable immune phenotype to the monoclonal a-PD-L1 mAb.

The discovery of a-PD-L1 therapy has been an important milestone in cancer therapy. However, existing therapies have limitations in efficacy and are associated with toxicity [70, 71]. The a-PD-L1 NP approach used in this study may be a platform to improve the therapeutic potential and reduce the toxicity of a-PD-L1 as well as other monoclonal antibody therapies.

\section{Material and Methods}

\section{Synthesis and Characterization of PD-LI F(ab) fragments}

Anti-mouse PD-L1 (clone 10F.9G2, BioXCell) antibody was fragmented using established protocols. Briefly, the whole IgG was digested with pepsin (ThermoFisher) according to manufacturer's instruction with minor modifications (16 hour incubation). The $\mathrm{F}(\mathrm{ab})_{2}$ fragment was collected by purifying the digest using a $50 \mathrm{kDa} \mathrm{MWCO}$ centrifuge filter (Vivaspin 500), and then further digested into $\mathrm{F}(\mathrm{ab})$ fragments with free sulfhydryl groups after incubation with TCEP $\mathrm{HCl}(20 \mathrm{mM})$ for 90 minutes. The $\mathrm{F}(\mathrm{ab})$ fragments were purified using 10K MWCO centrifuge filters. MALDI-TOF analysis confirmed the successful fragmentation of the antibody.

\section{a-PD-L1 F(ab)-PEG-PLGA Conjugation and Formation of Nanoparticles}

$\mathrm{F}(\mathrm{ab})$ fragments were coupled to MAL-PEG(5k)PLGA(5k) polymers (Nanosoft Polymers) prepared at $10 \mathrm{mg} / \mathrm{mL}$ in $0.1 \mathrm{M} \mathrm{NaPO}_{4}, 0.15 \mathrm{M} \mathrm{NaCl}$, and $10 \mathrm{mM}$ EDTA solution by incubating at a 1:10 $\mathrm{F}(\mathrm{ab})$ to PEG-PLGA ratio. The coupling was run at $4^{\circ} \mathrm{C}$ overnight before purification using 10K MWCO centrifuge filters. To form nanoparticles, a-PD-L1 $\mathrm{F}(\mathrm{ab})$-PEG-PLGA polymers were solvent-replaced with DMSO, and then added dropwise into ultra-pure water at 1:10 v/v ratio. The solution was then homogenized for 5 minutes to form a-PD-L1 F(ab)-PEG-PLGA nanoparticles. The nanoparticles were purified and concentrated using 10K MWCO centrifuge filters.

\section{Characterization of $\alpha$-PD-L1 F(ab)-PEG-PLGA Nanoparticles}

The particle size of a-PD-L1 NP was measured using Dynamic Light Scattering (DLS) method on a 90Plus Particle Size Analyzer (Brookhaven Instruments, Holtsville, NY) and Zetasizer Nano ZS90 (Malvern Panalytical). For stability experiments, data was collected at day $0,7,14,21,28,35$, and 70. Raw distribution data was plotted in GraphPad Prism software and fit using a Gaussian curve, with the mean being taken as the particle size for that replicate. The average of three separate replicates was taken to find the mean particle size \pm standard error of the mean (SEM). We also determined the zeta potential of the particles using a 90Plus Zeta Potential Analyzer (Brookhaven Instruments, Holtsville, NY) and Zetasizer Nano ZS90. Particle formulations were dissolved in DI water at 1:10 v/v. The average of three separate replicates was taken to find the mean zeta potential \pm SEM. The presence of equivalent amount of a-PD-L1 was verified through Nanodrop2000 and injected to mice in in vivo study.

\section{Cell Culture}

MC38 cells (generously donated by the laboratory of Dr. Ronald Levy) were cultured in T-75 flasks using DMEM supplemented with 10\% FBS, 100 units/ml penicillin/streptomycin, $2 \mathrm{mM}$ L-glutamine, and $0.1 \mathrm{mM}$ non-essential amino acids. The cells were maintained at $37^{\circ} \mathrm{C}, 5 \% \mathrm{CO} 2$, and $95 \%$ relative humidity. Cells were passaged at $80-90 \%$ confluency using a $0.25 \%$ trypsin $/ 0.20 \%$ EDTA solution. The cell doubling time was established to be 13 hours based on a series of time course cell counts.

\section{Animal Work}

Animals were housed in a pathogen-free 
environment at Stanford University and all procedures were performed in accordance with Stanford's Administrative Panel on Laboratory Animal Care (APLAC) protocols. For toxicity experiments and tumor development and therapy experiments, age and gender matched C57BL/6J mice (8-12 weeks) were utilized. For biodistribution experiments, NOD scid gamma (NSG) mice (4-6 weeks) were utilized.

\section{Toxicity}

For in vitro experiments, MC38 cells were cultured with $10 \mu \mathrm{g} / \mathrm{ml}$ of either a-PD-L1 mAb, a-PD-L1 NP, or empty NP and collected for cell count after approximately two doubling cycles. For in vivo experiments, $200 \mu \mathrm{g}$ of either control IgG, a-PD-L1 $\mathrm{mAb}$, or a-PD-L1 NP were given IP to healthy mice every 3 days for a total of 3 injections. Body weight and overall condition were recorded for 3-4 weeks. Spleens from healthy mice that received either PBS or empty NP were collected at 4 weeks and 8 weeks for examination of any unintended autoimmune toxicity from PEG-PLGA polymers.

\section{Biodistribution}

To measure the distribution of a-PD-L1 NP, NSG mice were administered with either $500 \mu \mathrm{g}$ of a-PD-L1 $\mathrm{mAb}$, an equivalent amount of a-PD-L1 NP, or other controls $\left(\mathrm{F}(\mathrm{ab})_{2}, \mathrm{~F}(\mathrm{ab})\right.$, and empty NP) fluorescently-labeled with $\mathrm{Cy} 5$ dye intravenously and imaged using an IVIS Spectrum fluorescence imager (Perkin Elmer) to trace the geographical distribution of the treatments over time. Fluorescence was measured at times $0,4,8$, and 24 hours, and organs were excised to measure fluorescence after 24 hours. This was confirmed after excising vital organs and measuring the fluorescence - the liver, kidneys, and GI all exhibit fluorescent intensity in the a-PD-L1 NP group versus all other control groups.

\section{Immunohistochemistry staining}

Spleens were harvested from age and gender matched C57BL/ 6 mice at three weeks post MC38 injection and fixed in $10 \%$ formalin $\mathrm{w} / \mathrm{v}(4 \%$ formaldehyde solution) for 48 hours at room temperature. The tissues were then stored in $70 \%$ ethanol until processing for paraffin embedding and sectioning. CD19 (1:800 in 5\% skim milk; Cell Signaling, \#90176T), CD4 (1:1000 in 5\% skim milk; abcam, \#ab183685), PD-L1 (1:300 in 1x TBS; Proteintech, \#17952-1-AP) were used in IHC according to manufacturer's protocol.

\section{Flow cytometry}

Spleens and tumors were harvested from C57BL/6 mice at 4 weeks following orthotopic transplantation with the MC38 colon adenocarcinoma cell line. Spleens were minced with fine scissors prior to passage through a $70 \mu \mathrm{M}$ nylon mesh filter (fisher scientific, 22363548) and washed with RPMI media (Corning, 21-040-CM). Red blood cells were lysed with ACK lysis buffer (Gibco, A10492-01) prior to resuspension in 1X PBS (Corning, 21-040-CV). Tumors were digested and processed according to manufacturer protocol using a Miltenyi tumor dissociation kit (130-096-730). Tumor cells were agitated after vigorous vortexing at RT in a 37C water bath for 30-60 min prior to washing with RPMI and straining through a mesh filter. Spleen and tumor cells were resuspended in $1 \mathrm{ml} 1 \mathrm{X}$ PBS at $0.5-1$ million cells and stained using a Live/Dead fixable near-IR dead cell stain kit fixable at 1:1000 dilution (Invitrogen, L34975). Cells were subsequently washed $2 x$ with PBS, resuspended in $100 \mu \mathrm{l}$ FACS buffer (PBS supplemented with $2 \%$ FBS and $0.5 \mathrm{mM}$ EDTA) and transferred to a U-bottom microtiter plate for staining. Fc block (BD Pharmingen, 553132) was added at 1:50 dilution and cells were incubated for $20 \mathrm{~min}$ on Ice followed by addition of $50 \mu \mathrm{l}$ of the prepared antibody cocktail (supplementary table 1). Cells were incubated with antibodies for $30 \mathrm{~min}$ on ice, washed twice with $200 \mu 1$ FACS buffer. The cells were resuspended in $100 \mu 1$ 1x fixation/permeabilization buffer ( BD Pharmingen) for 30 min on ice in the dark, washed $2 x$ with 1X permeabilization buffer ( BD Pharmingen) prior to resuspension in $200 \mu$ FACS buffer for flow cytometry analysis. Invitrogen UltraComp beads (01-2222-42) were prepared per manufacturer instructions and used to set up compensation. See Supplementary Table 1 for additional information.

\section{Tumor Development and Therapy}

C57BL/6 mice were injected subcutaneously with $0.5 \times 10^{6}$ or $0.1 \times 10^{6}$ MC38 cells in $100 \mu \mathrm{PBS}$ in their right flank and observed every day for palpable tumors. Animals were monitored every 3 days once tumor mass was detected. Body weight, tumor size, and overall condition were recorded. Animals were randomly enrolled into study groups (control IgG, a-PD-L1 mAb, or a-PD-L1 NP) and treatment begun 3-7 days based on the number of MC38 cells injected. $200 \mu \mathrm{g}$ of control IgG, a-PD-L1 mAb, or a-PD-L1 NP was injected in final volume of $100 \mu 1$ either IP or IV (based on the study objective) every 3 days for total of 3 injections. Palpable tumors were measured using calipers. Tumor volume was calculated $V=\Pi / 6 \mathrm{x}$ (length) $\mathrm{x}$ (width) $\mathrm{x}$ (height). When any of the tumor dimension reached $\geq 17 \mathrm{~mm}$ or mice lost more than $20 \%$ of their body weight, they were euthanized accordingly to protocol. Plasma, spleen, and tumor were collected for immunohistochemistry and flow 
cytometry.

\section{Statistical Analysis}

Results (mean \pm SEM) were analyzed for statistical significance by Student's t-test and one-way ANOVA using GraphPad Prism (Graphpad Software, Inc.). Significance is denoted by * for $\mathrm{p}<0.05$, ** for $\mathrm{p}<0.01$, and $* * *$ for $\mathrm{p}<0.001$.

\section{Supplementary Material}

Supplementary figures and table.

http://www.ntno.org/v06p0243s1.pdf

\section{Acknowledgements}

We dedicate this paper to the memory of Dr. Sanjiv Sam Gambhir, our friend, colleague and mentor. Thank you to Dr. Ronald Levy for the MC38 tumor cell line, the Stanford Canary Center and Dr. Gambhir lab for their help in running MALDI-TOF analysis and Zetasizer Nano ZS90. Flow cytometry for this project was performed with the Symphony and LSRII Analysers in the Stanford Shared FACS Facility using a NIH S10 Shared Instrument Grant (Symphony 1S10OD026831-01). We would also like to thank the members of the Felsher lab for their helpful comments. Part of this work was performed at the Stanford Nano Shared Facilities (SNSF) and supported by the Translational Research and Applied Medicine (TRAM) at Stanford Medicine and NIH grant SIG\#1S10RR02678001. Dr. Lee and Dr. Kuruvilla were supported by NIH T32 Cancer Translational Nanotechnology Training (TNT) grant (5T32CA196585) and Dr. Atibalentja by NHLBI Hematology T32 (T32HL120824). Dr. Felsher receives support from the NIH (grants CA208735, CA253180, CA188383 and CA184384).

\section{Competing Interests}

The authors have declared that no competing interest exists.

\section{References}

1. Kato K, et al. KEYNOTE-590: Phase III study of first-line chemotherapy with or without pembrolizumab for advanced esophageal cancer. Future Oncol, 2019; 15(10): 1057-1066.

2. Larkin J, et al. Combined Nivolumab and Ipilimumab or Monotherapy in Untreated Melanoma. N Engl J Med, 2015; 373(1): 23-34

3. Sezer A, et al. Cemiplimab monotherapy for first-line treatment of advanced non-small-cell lung cancer with PD-L1 of at least 50\%: a multicentre, open-label, global, phase 3, randomised, controlled trial. Lancet, 2021; 397(10274): 592-604.

4. Rittmeyer A, et al. Atezolizumab versus docetaxel in patients with previously treated non-small-cell lung cancer $(\mathrm{OAK})$ : a phase 3, open-label, multicentre randomised controlled trial. Lancet, 2017; 389(10066): 255-265.

5. Powles T, et al. Avelumab Maintenance Therapy for Advanced or Metastatic Urothelial Carcinoma. N Engl J Med, 2020; 383(13): 1218-1230.

6. Ascierto PA, et al. Ipilimumab $10 \mathrm{mg} / \mathrm{kg}$ versus ipilimumab $3 \mathrm{mg} / \mathrm{kg}$ in patients with unresectable or metastatic melanoma: a randomised, double-blind, multicentre, phase 3 trial. Lancet Oncol, 2017; 18(5): 611-622.

7. Sharma $\mathrm{P}$, et al. Primary, Adaptive, and Acquired Resistance to Cancer Immunotherapy. Cell, 2017; 168(4): 707-723.
8. Hellmann MD, et al. Nivolumab plus Ipilimumab in Lung Cancer with a High Tumor Mutational Burden. N Engl J Med, 2018; 378(22): 2093-2104.

9. Capdevila J, et al. PD-1 Blockade in Anaplastic Thyroid Carcinoma. J Clin Oncol, 2020. 38(23): 2620-2627.

10. Spain L, et al. Management of toxicities of immune checkpoint inhibitors. Cancer Treat Rev, 2016. 44: 51-60.

11. Friedman $\mathrm{CF}$, et al. Treatment of the Immune-Related Adverse Effects of Immune Checkpoint Inhibitors: A Review. JAMA Oncol, 2016. 2(10): 1346-1353.

12. Rizvi NA, et al. Durvalumab With or Without Tremelimumab vs Standard Chemotherapy in First-line Treatment of Metastatic Non-Small Cell Lung Cancer: The MYSTIC Phase 3 Randomized Clinical Trial. JAMA Oncol, 2020. 6(5): 661-674.

13. Thurber GM, et al. Antibody tumor penetration: transport opposed by systemic and antigen-mediated clearance. Adv Drug Deliv Rev, 2008. 60(12): 1421-34.

14. Sharkey RM, et al. Biodistribution and radiation dose estimates for yttriumand iodine-labeled monoclonal antibody IgG and fragments in nude mice bearing human colonic tumor xenografts. Cancer Res, 1990. 50(8): 2330-6.

15. Sher AF, et al. Fatal Adverse Events Associated with Pembrolizumab in Cancer Patients: A Meta-Analysis. Cancer Invest, 2020. 38(2): 130-138.

16. Wang DY, et al. Fatal Toxic Effects Associated With Immune Checkpoint Inhibitors: A Systematic Review and Meta-analysis. JAMA Oncol, 2018. 4(12): 1721-1728.

17. Tanios GE, et al. Autoimmune hemolytic anemia associated with the use of immune checkpoint inhibitors for cancer: 68 cases from the Food and Drug Administration database and review. Eur J Haematol, 2019. 102(2): 157-162.

18. Safa $\mathrm{H}$, et al. Immune checkpoint inhibitor related myasthenia gravis: single center experience and systematic review of the literature. J Immunother Cancer, 2019. 7(1): 319.

19. Illum $L$, et al. Tissue distribution of poly(hexyl 2-cyanoacrylate) nanoparticles coated with monoclonal antibodies in mice bearing human tumor xenografts. J Pharmacol Exp Ther, 1984. 230(3): 733-6.

20. Wang $W$, et al. Monoclonal antibody pharmacokinetics and pharmacodynamics. Clin Pharmacol Ther, 2008. 84(5): 548-58.

21. Stephenson KE, et al. Immunogenicity of the Ad26.COV2.S Vaccine for COVID-19. JAMA, 2021. 325(15): 1535-1544.

22. Jazayeri JA and Carroll GJ. Fc-based cytokines: prospects for engineering superior therapeutics. BioDrugs, 2008. 22(1): 11-26.

23. Catenacci DVT, et al. Margetuximab plus pembrolizumab in patients with previously treated, HER2-positive gastro-oesophageal adenocarcinoma (CP-MGAH22-05): a single-arm, phase 1b-2 trial. Lancet Oncol, 2020. 21(8): 1066-1076

24. Martins F, et al. Adverse effects of immune-checkpoint inhibitors: epidemiology, management and surveillance. Nat Rev Clin Oncol, 2019. 16(9): 563-580.

25. Bapatla A, et al. Atezolizumab, a PD-L1 Inhibitor: An Association of Bleeding Gastric Ulcer With Its Use. Cureus, 2021. 13(6): p. e15637.

26. Tian $Y$, et al. Immune Checkpoint Inhibitors-Induced Hepatitis. Adv Exp Med Biol, 2018. 995: 159-164

27. Khunger M, et al. Incidence of Pneumonitis With Use of Programmed Death 1 and Programmed Death-Ligand 1 Inhibitors in Non-Small Cell Lung Cancer: A Systematic Review and Meta-Analysis of Trials. Chest, 2017. 152(2): 271-281.

28. Veronese FM and Mero A. The impact of PEGylation on biological therapies. BioDrugs, 2008. 22(5): 315-29.

29. Veronese FM and Pasut G. PEGylation, successful approach to drug delivery. Drug Discov Today, 2005. 10(21): 1451-8.

30. Rafiei P and Haddadi A. Docetaxel-loaded PLGA and PLGA-PEG nanoparticles for intravenous application: pharmacokinetics and biodistribution profile. Int J Nanomedicine, 2017. 12: 935-947.

31. Barenholz Y. Doxil®--the first FDA-approved nano-drug: lessons learned. J Control Release, 2012. 160(2): 117-34.

32. Sadat Tabatabaei Mirakabad F, et al. PLGA-based nanoparticles as cancer drug delivery systems. Asian Pac J Cancer Prev, 2014. 15(2): 517-35.

33. Danhier F, et al. PLGA-based nanoparticles: an overview of biomedical applications. J Control Release, 2012. 161(2): 505-22.

34. Makadia HK and Siegel SJ. Poly Lactic-co-Glycolic Acid (PLGA) as Biodegradable Controlled Drug Delivery Carrier. Polymers (Basel), 2011. 3(3): 1377-1397.

35. Swain S, et al. Nanoparticles for Cancer Targeting: Current and Future Directions. Curr Drug Deliv, 2016. 13(8): 1290-1302.

36. Davis ME. The first targeted delivery of siRNA in humans via a self-assembling, cyclodextrin polymer-based nanoparticle: from concept to clinic. Mol Pharm, 2009. 6(3): 659-68.

37. Mitchell MJ, et al. Engineering precision nanoparticles for drug delivery. Nat Rev Drug Discov, 2021. 20(2): 101-124.

38. Champion JA and Mitragotri S. Role of target geometry in phagocytosis. Proc Natl Acad Sci U S A, 2006. 103(13): 4930-4.

39. Bocci V. Evaluation of routes of administration of interferon in cancer: a review and a proposal. Cancer Drug Deliv, 1984. 1(4): 337-51.

40. Nierkens S, et al. Route of administration of the TLR9 agonist CpG critically determines the efficacy of cancer immunotherapy in mice. PLoS One, 2009. 4(12): e8368.

41. Marabelle A, et al. Intratumoral immunotherapy: using the tumor as the remedy. Ann Oncol, 2017. 28(suppl_12): xii33-xii43. 
42. Lin $\mathrm{H}$, et al. Host expression of PD-L1 determines efficacy of PD-L1 pathway blockade-mediated tumor regression. J Clin Invest, 2018. 128(4): 1708.

43. Hartley GP, et al. Programmed Cell Death Ligand 1 (PD-L1) Signaling Regulates Macrophage Proliferation and Activation. Cancer Immunol Res, 2018. 6(10): 1260-1273.

44. Wu Y, et al. PD-L1 Distribution and Perspective for Cancer Immunotherapy-Blockade, Knockdown, or Inhibition. Front Immunol, 2019. 10: 2022.

45. Cha JH, et al. Mechanisms Controlling PD-L1 Expression in Cancer. Mol Cell, 2019. 76(3): 359-370.

46. Rempel SA, et al. Splenic and immune alterations of the Sparc-null mouse accompany a lack of immune response. Genes Immun, 2007. 8(3): 262-74.

47. Bartolomé-Izquierdo $\mathrm{N}$, et al. miR-28 regulates the germinal center reaction and blocks tumor growth in preclinical models of non-Hodgkin lymphoma. Blood, 2017. 129(17): 2408-2419.

48. Coppola D, et al. Unique ectopic lymph node-like structures present in human primary colorectal carcinoma are identified by immune gene array profiling. Am J Pathol, 2011. 179(1): 37-45.

49. Gubin MM, et al. High-Dimensional Analysis Delineates Myeloid and Lymphoid Compartment Remodeling during Successful Immune-Checkpoint Cancer Therapy. Cell, 2018. 175(4): 1014-1030.e19.

50. Kiss M, et al. Myeloid cell heterogeneity in cancer: not a single cell alike. Cell Immunol, 2018. 330: 188-201.

51. Kadkhoda J, et al. Advances in antibody nanoconjugates for diagnosis and therapy: A review of recent studies and trends. Int J Biol Macromol, 2021. 185: 664-678.

52. Capolla S, et al. Targeted tumor imaging of anti-CD20-polymeric nanoparticles developed for the diagnosis of B-cell malignancies. Int J Nanomedicine, 2015. 10: 4099-109.

53. Khaw BA, et al. Bispecific antibody complex pre-targeting and targeted delivery of polymer drug conjugates for imaging and therapy in dual human mammary cancer xenografts: targeted polymer drug conjugates for cancer diagnosis and therapy. Eur J Nucl Med Mol Imaging, 2014. 41(8): 1603-16.

54. Sumer Bolu B, et al. Trastuzumab targeted micellar delivery of docetaxel using dendron-polymer conjugates. Biomater Sci, 2020. 8(9): 2600-2610.

55. Eggermont AM, et al. Long-term results of the randomized phase III trial EORTC 18991 of adjuvant therapy with pegylated interferon alfa-2b versus observation in resected stage III melanoma. J Clin Oncol, 2012. 30(31): 3810-8.

56. Eggermont AMM, et al. Adjuvant therapy with pegylated interferon-alfa2b vs observation in stage II B/C patients with ulcerated primary: Results of the European Organisation for Research and Treatment of Cancer 18081 randomised trial. Eur J Cancer, 2020. 133: 94-103.

57. Kumar D, et al. Antibody-targeted nanoparticles for cancer treatment, in NanoBioMedicine. 2020. 35-65.

58. Petrilli $\mathrm{R}$, et al. Immunoconjugates for Cancer Targeting: A Review of Antibody-Drug Conjugates and Antibody-Functionalized Nanoparticles. Curr Med Chem, 2021. 28(13): 2485-2520.

59. Ovacik M and Lin K. Tutorial on Monoclonal Antibody Pharmacokinetics and Its Considerations in Early Development. Clin Transl Sci, 2018. 11(6): 540-552.

60. Cataldi M, et al. Emerging Role of the Spleen in the Pharmacokinetics of Monoclonal Antibodies, Nanoparticles and Exosomes. Int J Mol Sci, 2017. 18(6).

61. Poon W, et al. Elimination Pathways of Nanoparticles. ACS Nano, 2019. 13(5): 5785-5798.

62. Du B, et al. Glomerular barrier behaves as an atomically precise bandpass filter in a sub-nanometre regime. Nat Nanotechnol, 2017. 12(11): 1096-1102.

63. Dölen $Y$, et al. Nanovaccine administration route is critical to obtain pertinent iNKt cell help for robust anti-tumor T and B cell responses. Oncoimmunology, 2020. 9(1): 1738813.

64. McLennan DN, et al. Subcutaneous drug delivery and the role of the lymphatics. Drug Discov Today Technol, 2005. 2(1): 89-96.

65. Hickey JW, et al. Control of polymeric nanoparticle size to improve therapeutic delivery. J Control Release, 2015. 219: 536-547.

66. Peng $Q$, et al. PD-L1 on dendritic cells attenuates $T$ cell activation and regulates response to immune checkpoint blockade. Nat Commun, 2020. 11(1): 4835.

67. Fransen MF, et al. Tumor-draining lymph nodes are pivotal in PD-1/PD-L1 checkpoint therapy. JCI Insight, 2018. 3(23).

68. Candeias SM and Gaipl US. The Immune System in Cancer Prevention, Development and Therapy. Anticancer Agents Med Chem, 2016. 16(1): 101-7.

69. Darvin $P$, et al. Immune checkpoint inhibitors: recent progress and potential biomarkers. Exp Mol Med, 2018. 50(12): 1-11.

70. Lin $X$, et al. Progress in PD-1/PD-L1 pathway inhibitors: From biomacromolecules to small molecules. Eur J Med Chem, 2020. 186: p. 111876.

71. Xia L, et al. PD-1/PD-L1 Blockade Therapy in Advanced Non-Small-Cell Lung Cancer: Current Status and Future Directions. Oncologist, 2019. 24(Suppl 1): S31-S41. 